\title{
Characterizations of the spectral radius of nonnegative weakly irreducible tensors via digraph
}

\author{
Lizhu Sun ${ }^{\mathrm{a}}$, Baodong Zheng ${ }^{\mathrm{a}}$, Yimin $\mathrm{Wei}^{\mathrm{b}}$, Changjiang $\mathrm{Bu}^{\mathrm{c}}$ \\ ${ }^{a}$ School of Science, Harbin Institute of Technology, Harbin 150001, PR China \\ ${ }^{b}$ School of Mathematical Sciences and Shanghai Key Laboratory of Contemporary Applied \\ Mathematics, Fudan University, Shanghai, 200433, PR China \\ ${ }^{c}$ College of Science, Harbin Engineering University, Harbin 150001, PR China
}

\begin{abstract}
For a nonnegative weakly irreducible tensor $\mathcal{A}$, we give some characterizations of the spectral radius of $\mathcal{A}$, by using the digraph of tensors. As applications, some bounds on the spectral radius of the adjacency tensor and the signless Laplacian tensor of the $k$-uniform hypergraphs are shown.
\end{abstract}

Keywords: Nonnegative tensor, Spectral radius, Digraph, $k$-uniform hypergraph AMS classification: 15A69, 15A18

\section{Introduction}

An order $m$ dimension $n$ tensor $\mathcal{A}$ consist of $n^{m}$ complex entries,

$$
\mathcal{A}=\left(a_{i_{1} i_{2} \cdots i_{m}}\right), 1 \leqslant i_{j} \leqslant n(j=1,2, \ldots, m) .
$$

The tensor $A=\left(a_{i_{1} i_{2} \cdots i_{m}}\right)$ is called symmetric if $a_{i_{1} i_{2} \cdots i_{m}}=a_{\sigma\left(i_{1}\right) \sigma\left(i_{2}\right) \cdots \sigma\left(i_{m}\right)}$, where $\sigma$ is any permutation of the indices. The tensor $\mathcal{A}$ is called nonnegative if all the entries $a_{i_{1} \ldots i_{m}} \geqslant 0$. Let $\mathbb{R}_{+}^{[m, n]}$ be the set of order $m$ dimension $n$ nonnegative tensors. And let $\mathbb{R}_{++}^{n}$ be the set of the dimension $n$ positive vectors (all the entries positive).

In 2005, the eigenvalue of tensors is defined by Qi [15] and Lim [11], respectively. For a complex tensor $\mathcal{A}$ of order $m$ dimension $n$, a complex

Email addresses: sunlizhu678876@126.com (Lizhu Sun), zbd@hit.edu.cn (Baodong Zheng), ymwei@fudan.edu.cn (Yimin Wei), buchangjiang@hrbeu.edu.cn (Changjiang $\mathrm{Bu}$ ) 
number $\lambda$ and a nonzero complex vector $x=\left(x_{1}, x_{2}, \ldots, x_{n}\right)^{\mathrm{T}}$ is called an eigenvalue and an eigenvector (corresponding to $\lambda$ ) of the tensor $\mathcal{A}$, respectively, if they satisfy

$$
\mathcal{A} x^{m-1}=\lambda x^{[m-1]},
$$

where $\mathcal{A} x^{m-1}$ is a dimension $n$ vector with entry

$$
\left(\mathcal{A} x^{m-1}\right)_{i}=\sum_{i_{2}, \ldots, i_{m}=1}^{n} a_{i i_{2} \ldots i_{m}} x_{i_{2}} \ldots x_{i_{m}}, i=1,2, \ldots, n
$$

and $x^{[m-1]}=\left(x_{1}^{m-1}, x_{2}^{m-1}, \ldots, x_{n}^{m-1}\right)^{\mathrm{T}}($ see [15]). Let $\rho(\mathcal{A})=\sup \{|\lambda|: \lambda \in$ $\operatorname{spec}(\mathcal{A})\}$ be the spectral radius of tensor $\mathcal{A}$, where $\operatorname{spec}(\mathcal{A})$ is the set of all the eigenvalues of $\mathcal{A}$.

Recently, the spectral theory of tensors has attracted much attention [3, 5, 6, 19, 20]. In 2005, Lim [11] proposes the definition of irreducible tensors. In 2008, Chang et al. [5] give the Perron-Frobenius Theorem for nonnegative irreducible tensors. It is shown that $\rho(\mathcal{A})$ is an eigenvalue of nonnegative irreducible tensors, and $\rho(\mathcal{A})$ is the only eigenvalue with nonnegative eigenvectors [5]. Similarly as the Collatz-Wielandt Theorem of matrices, the Minimax Theorem of the spectral radius of nonnegative irreducible tensors $\mathcal{A}$ is given as follows (see [5])

$$
\min _{x \in \mathbb{R}_{++}^{n}} \max _{1 \leqslant i \leqslant n} \frac{\left(\mathcal{A} x^{m-1}\right)_{i}}{x_{i}^{m-1}}=\rho(\mathcal{A})=\max _{x \in \mathbb{R}_{++}^{n}} \min _{1 \leqslant i \leqslant n} \frac{\left(\mathcal{A} x^{m-1}\right)_{i}}{x_{i}^{m-1}} .
$$

Scholars pay much attention to find the largest eigenvalue of nonnegative irreducible tensors [7, 12, 13, 21]. The weakly irreducible tensors are defined by associated with tensors a digraph [8, 14].

Definition 1.1. An order $m$ dimension $n$ tensor $\mathcal{A}$ over real field is called weakly irreducible if the digraph $G_{\mathcal{A}}$ is strongly connected.

Digraph $G_{\mathcal{A}}$ and the strongly connectivity are introduced in Section 2. If tensor $\mathcal{A}$ is irreducible then it is weakly irreducible [8, 14]. For the nonnegative weakly irreducible tensor $\mathcal{A}$, there exists a positive eigenvector corresponding to the eigenvalue $\rho(\mathcal{A})$ (see [8]).

A hypergraph $\mathcal{H}=(V(\mathcal{H}), E(\mathcal{H}))$ is called $k$-uniform if each edge of $\mathcal{H}$ contains exactly $k$ distinct vertices [4]. The adjacency tensor of $\mathcal{H}$, denoted by $\mathcal{A}_{\mathcal{H}}=\left(a_{i_{1} i_{2} \cdots i_{k}}\right)$, is an order $k$ dimension $|V(\mathcal{H})|$ tensor with entries

$$
a_{i_{1} i_{2} \cdots i_{k}}=\left\{\begin{array}{cc}
\frac{1}{(k-1) !}, & \left\{i_{1} i_{2} \cdots i_{k}\right\} \in E(\mathcal{H}), \\
0, & \text { otherwises }
\end{array}\right.
$$


The degree tensor of the $k$-uniform uniform hypergraph $\mathcal{H}$, denoted by $\mathcal{D}_{\mathcal{H}}$, is an order $k$ dimension $|V(\mathcal{H})|$ diagonal tensor whose $(i \cdots i)$-diagonal entry is the degree of vertex $i, i=1,2, \ldots,|V(\mathcal{H})|$. The tensor $\mathcal{Q}_{\mathcal{H}}=$ $\mathcal{D}_{\mathcal{H}}+\mathcal{A}_{\mathcal{H}}$ is called the signless Laplacian tensor of the $\mathcal{H}$. Recently, the spectral theory of hypergraphs developed rapidly [4, 9, 16, 18, 22].

It is well-known that the nonnegative irreducible matrices are closely related to the digraphs [1]. For a nonnegative irreducible matrices $M$ with all diagonal entries zero, Brualdi [2] gives the characterizations of the spectral radius of $M$ by using the associated digraph.

In this paper, we use the digraph of tensors to characterize the spectral radius of the nonnegative weakly irreducible tensors, which generalize the results of matrices to tensors [2]. By applying the characterizations, some bounds on the spectral radius of the adjacency tensor and signless Laplacian tensor of a $k$-uniform hypergraph are shown.

\section{Preliminary}

For an order $m$ dimension $n$ tensor $A=\left(a_{i_{1} i_{2} \cdots i_{m}}\right)$, let $G_{\mathcal{A}}=\left(V\left(G_{\mathcal{A}}\right), E\left(G_{\mathcal{A}}\right)\right)$ be the digraph of the tensor $\mathcal{A}$ with vertex set $V\left(G_{\mathcal{A}}\right)=\{1,2, \ldots, n\}$ and arc set $E\left(G_{\mathcal{A}}\right)=\left\{(i, j) \mid a_{i i_{2} \ldots i_{m}} \neq 0, j \in\left\{i_{2}, \ldots, i_{m}\right\}\right\}$ (see [8, 14]). If there exist directed paths from $i$ to $j$ and $j$ to $i$ for each $i, j \in V\left(G_{\mathcal{A}}\right)(i \neq j)$, then $G_{\mathcal{A}}$ is called strongly connected. Denote the set of the circuits in $G_{\mathcal{A}}$ by $C\left(G_{\mathcal{A}}\right)$ (Loops in the circuits are allowed). Let $G_{\mathcal{A}}^{+}(v):=\left\{u \in V\left(G_{\mathcal{A}}\right):(v, u) \in E\left(G_{\mathcal{A}}\right)\right\}$.

Define a map $f$ from the vertex set of $G_{\mathcal{A}}$ to the real field, $f: V\left(G_{\mathcal{A}}\right) \rightarrow \mathbb{R}$, $f$ is called a vertex labelling of $G_{\mathcal{A}}$. By the Lemma 2.6 of [2], we can get the following result.

Lemma 2.1. [2] Let $G_{\mathcal{A}}$ be the digraph of $\mathcal{A}$ with a vertex labelling $f$ on $V\left(G_{\mathcal{A}}\right)$. If $G_{\mathcal{A}}^{+}(v)$ is nonempty for each $v \in V\left(G_{\mathcal{A}}\right)$, then there exist circuits $\left\{v_{i_{1}}, v_{i_{2}}, \ldots, v_{i_{k}}, v_{i_{k+1}}\right.$

$\left.=v_{i_{1}}\right\}$ and $\left\{v_{t_{1}}, v_{t_{2}}, \ldots, v_{t_{s}}, v_{t_{s+1}}=v_{t_{1}}\right\}$ (Loops in the circuits are allowed) such that $f\left(v_{i_{j+1}}\right)=\max \left\{f(v): v \in G_{\mathcal{A}}^{+}\left(v_{i_{j}}\right)\right\}$ and $f\left(v_{t_{l+1}}\right)=\min \{f(v): v \in$ $\left.G_{\mathcal{A}}^{+}\left(v_{t_{l}}\right)\right\} j=1,2, \ldots, k, l=1,2, \ldots, s$, respectively.

Lemma 2.2. [8] Let $\mathcal{A} \in \mathbb{R}_{+}^{[m, n]}$ be a weakly irreducible tensor. Then $\rho(\mathcal{A})$ is an eigenvalue of $\mathcal{A}$, and there exists a unique positive eigenvector corresponding to $\rho(\mathcal{A})$ up to a multiplicative constant. 
Lemma 2.3. [17] For an order $m$ dimension $n$ tensor $\mathcal{A}=\left(a_{i_{1} i_{2} \ldots i_{m}}\right)$ and an invertible diagonal matrix $D=\operatorname{diag}\left(d_{11}, d_{22}, \ldots, d_{n n}\right), \mathcal{B}=D^{-(m-1)} \mathcal{A} D$ is an order $m$ dimension $n$ tensor with entries

$$
b_{i_{1} i_{2} \ldots i_{m}}=d_{i_{1} i_{1}}^{-(m-1)} a_{i_{1} i_{2} \ldots i_{m}} d_{i_{2} i_{2}} \cdots d_{i_{m} i_{m}} .
$$

In this case, $\mathcal{A}$ and $\mathcal{B}$ are called diagonal similar, and $\mathcal{A}$ and $\mathcal{B}$ have the same spectrum.

Lemma 2.4. 14] Let $\mathcal{H}$ be an k-uniform hypergraph. Then $\mathcal{A}_{\mathcal{H}} \in \mathbb{R}_{+}^{[m, n]}$ (and $\mathcal{Q}_{\mathcal{H}} \in \mathbb{R}_{+}^{[m, n]}$ ) is weakly irreducible if and only if $\mathcal{H}$ is connected.

\section{Main results}

For a tensor $\mathcal{A}=\left(a_{i_{1} i_{2} \cdots i_{m}}\right) \in \mathbb{R}_{+}^{[m, n]}$, we denote the sum of $i$-th slice of $\mathcal{A}$ by $K_{i}=\sum_{i_{2}, \ldots, i_{m}=1}^{n} a_{i i_{2} \cdots i_{m}}, i=1,2, \ldots, n$. Let $|\gamma|$ be the length of the circuit $\gamma \in C\left(G_{\mathcal{A}}\right)$.

We first give two results on the bounds of spectral radius for nonnegative weakly irreducible tensors, which extend Theorem 4.7 and Corollary 4.6, 4.8 of [2] to tensors.

Theorem 3.1. Let $\mathcal{A}=\left(a_{i_{1} i_{2} \ldots i_{m}}\right) \in \mathbb{R}_{+}^{[m, n]}$ be a weakly irreducible tensor. Then

$$
\min _{\gamma \in C\left(G_{\mathcal{A}}\right)}\left(\prod_{i \in \gamma} K_{i}\right)^{\frac{1}{|\gamma|}} \leqslant \rho(\mathcal{A}) \leqslant \max _{\gamma \in C\left(G_{\mathcal{A}}\right)}\left(\prod_{i \in \gamma} K_{i}\right)^{\frac{1}{|\gamma|}} .
$$

Proof. By Lemma 2.2, we suppose that $x=\left(x_{1}, x_{2}, \ldots, x_{n}\right)^{\mathrm{T}}$ is a positive eigenvector corresponding to the eigenvalue $\rho(\mathcal{A})$. Let $f(i)=x_{i}\left(i \in V\left(G_{\mathcal{A}}\right)\right)$ be the vertex labelling of digraph $G_{\mathcal{A}}$. From Definition 1.1, we have $G_{\mathcal{A}}^{+}(i)$ is nonempty for each $i \in V\left(G_{\mathcal{A}}\right)$. Lemma 2.1 gives that there exists at least one circuit $\gamma_{1}=\left\{i_{1}, i_{2}, \ldots, i_{p}, i_{p+1}=i_{1}\right\}$ such that $x_{i_{j+1}} \geqslant x_{k}$, for each $k \in G_{\mathcal{A}}^{+}\left(i_{j}\right), j=1,2, \ldots, p$. Hence,

$$
\begin{aligned}
\rho(\mathcal{A}) x_{i_{j}}^{m-1} & =\sum_{k_{2}, \cdots, k_{m}=1}^{n} a_{i_{j} k_{2} \ldots k_{m}} x_{k_{2}} \cdots x_{k_{m}} \\
& \leqslant\left(\sum_{k_{2}, \cdots, k_{m}=1}^{n} a_{i_{j} k_{2} \ldots k_{m}}\right) x_{i_{j+1}^{m-1}}^{m-1} \\
& =K_{i_{j}} x_{i_{j+1}}^{m-1},
\end{aligned}
$$


for $j=1,2, \ldots, p$. Thus

$$
(\rho(\mathcal{A}))^{p} \prod_{j=1}^{p} x_{i_{j}}^{m-1} \leqslant \prod_{j=1}^{p} K_{i_{j}} x_{i_{j+1}}^{m-1}
$$

Note that $x=\left(x_{1}, x_{1}, \ldots, x_{n}\right)^{\mathrm{T}}$ is positive, so we can get

$$
\rho(\mathcal{A}) \leqslant\left(\prod_{j=1}^{p} K_{i_{j}}\right)^{\frac{1}{p}}
$$

that is

$$
\rho(\mathcal{A}) \leqslant\left(\prod_{i \in \gamma_{1}} K_{i}\right)^{\frac{1}{p}}
$$

Lemma 2.1 also shows that there exists at least one circuit $\gamma_{2}=\left\{v_{t_{1}}, v_{t_{2}}, \ldots, v_{t_{s}}, v_{t_{s+1}}=\right.$ $\left.v_{t_{1}}\right\}$ such that $x_{t_{l+1}} \leqslant x_{k}$ for each $k \in G_{\mathcal{A}}^{+}\left(t_{l}\right), l=1,2, \ldots, s$. Similarly as the above proof, we can get

$$
\rho(\mathcal{A}) \geqslant\left(\prod_{i \in \gamma_{2}} K_{i}\right)^{\frac{1}{s}}
$$

Thus

$$
\min _{\gamma \in C\left(G_{\mathcal{A}}\right)}\left(\prod_{i \in \gamma} K_{i}\right)^{\frac{1}{|\gamma|}} \leqslant \rho(\mathcal{A}) \leqslant \max _{\gamma \in C\left(G_{\mathcal{A}}\right)}\left(\prod_{i \in \gamma} K_{i}\right)^{\frac{1}{|\gamma|}}
$$

Remark. It is easy to see that if $K_{1}=K_{2}=\cdots=K_{n}$, the equalities in the above theorem hold.

Lemma 2.4 gives that the adjacency tensor (and signless Laplacian tensor) of a connected hypergraph $\mathcal{A}_{\mathcal{H}}$ (and $\mathcal{Q}_{\mathcal{H}}$ ) is nonnegative weakly irreducible, so $\rho\left(\mathcal{A}_{\mathcal{H}}\right)$ (and $\rho\left(\mathcal{Q}_{\mathcal{H}}\right)$ ) is an eigenvalue of $\mathcal{A}_{\mathcal{H}}\left(\right.$ and $\left.\mathcal{Q}_{\mathcal{H}}\right)$. In [4], it is shown that the largest eigenvalue $\lambda_{\text {max }}$ of $\mathcal{A}_{\mathcal{H}}$ is between the maximum degree $d_{\max }$ and the average degree $\bar{d}$ of $\mathcal{H}, \bar{d} \leqslant \lambda_{\max } \leqslant d_{\max }$. By using Theorem 3.1 , we also show the bounds on the largest eigenvalue of adjacency tensor and signless Laplacian tensor in terms of the degrees. 
Theorem 3.2. Let $\mathcal{H}$ be a connected $k$-uniform hypergraph with $n$ vertices. Suppose that $d_{i}$ is the degree of vertex $i, i=1,2, \ldots, n$. Then

$$
\min _{\gamma \in C\left(G_{\mathcal{A}_{\mathcal{H}}}\right)}\left(\prod_{i \in \gamma} d_{i}\right)^{\frac{1}{|\gamma|}} \leqslant \rho\left(\mathcal{A}_{\mathcal{H}}\right) \leqslant \max _{\gamma \in C\left(\mathcal{A}_{\mathcal{H}}\right)}\left(\prod_{i \in \gamma} d_{i}\right)^{\frac{1}{|\gamma|}}
$$

and

$$
\min _{\gamma \in C\left(G_{\left.\mathcal{Q}_{\mathcal{H}}\right)}\right.}\left(\prod_{i \in \gamma} 2 d_{i}\right)^{\frac{1}{|\gamma|}} \leqslant \rho\left(\mathcal{Q}_{\mathcal{H}}\right) \leqslant \max _{\gamma \in C\left(\mathcal{Q}_{\mathcal{H}}\right)}\left(\prod_{i \in \gamma} 2 d_{i}\right)^{\frac{1}{|\gamma|}} .
$$

The shortest length of the circuits in $G_{\mathcal{A}}$ is called the girth of $G_{\mathcal{A}}$. If we bring an order to the slice sums $K_{i}(i=1,2, \ldots, n)$ of tensor $\mathcal{A}$, the following result can be obtained.

Theorem 3.3. Let $\mathcal{A}=\left(a_{i_{1} i_{2} \ldots i_{m}}\right) \in \mathbb{R}_{+}^{[m, n]}$ be a weakly irreducible tensor. Suppose that $K_{1} \leqslant K_{2} \leqslant \cdots \leqslant K_{n}$ and the girth of $G_{\mathcal{A}}$ is $\mathrm{g}$. Then

$$
\left(K_{1} K_{2} \cdots K_{g}\right)^{\frac{1}{g}} \leqslant \rho(\mathcal{A}) \leqslant\left(K_{n-g+1} K_{n-g+2} \cdots K_{n}\right)^{\frac{1}{g}} .
$$

Proof. By Theorem 3.1, we have there exist circuits $\gamma_{1}, \gamma_{2} \in C\left(G_{\mathcal{A}}\right)$ of length $\left|\gamma_{1}\right| \geqslant g,\left|\gamma_{2}\right| \geqslant g$ such that

$$
\left(\prod_{i \in \gamma_{2}} K_{i}\right)^{\frac{1}{\left|\gamma_{2}\right|}} \leqslant \rho(\mathcal{A}) \leqslant\left(\prod_{i \in \gamma_{1}} K_{i}\right)^{\frac{1}{\left|\gamma_{1}\right|}}
$$

Since

$$
\left(\prod_{i \in \gamma_{1}} K_{i}\right)^{\frac{1}{\left|\gamma_{1}\right|}} \leqslant\left(K_{n-\left|\gamma_{1}\right|+1} K_{n-\left|\gamma_{1}\right|+2} \cdots K_{n}\right)^{\frac{1}{\left|\gamma_{1}\right|}} \leqslant\left(K_{n-g+1} K_{n-g+2} \cdots K_{n}\right)^{\frac{1}{g}}
$$

and

$$
\left(\prod_{i \in \gamma_{2}} K_{i}\right)^{\frac{1}{\left|\gamma_{2}\right|}} \geqslant\left(K_{1} K_{2} \cdots K_{\left|\gamma_{2}\right|}\right)^{\frac{1}{\left|\gamma_{2}\right|}} \geqslant\left(K_{1} K_{2} \cdots K_{g}\right)^{\frac{1}{g}},
$$

we obtain the theorem holds.

For a connected $k$-uniform hypergraph $\mathcal{H}$, According to the definition of hypergraph, there are no loops in $G_{\mathcal{A}_{\mathcal{H}}}$. And since $\mathcal{A}_{\mathcal{H}}$ is symmetric tensor, we have the girth of $G_{\mathcal{A}_{\mathcal{H}}}$ is 2 . Clearly, the girth of $G_{\mathcal{Q}_{\mathcal{H}}}$ is 1 . Hence, it follows from Theorem 3.2 and 3.3 that the follow result can be obtained, which are shown in [4] and [10], respectively. 
Corollary 3.4. For a connected $k$-uniform hypergraph $\mathcal{H}$, if $\mathcal{H}$ is regular with degree $d$, then $\rho\left(\mathcal{A}_{\mathcal{H}}\right)=d$ and $\rho\left(\mathcal{Q}_{\mathcal{H}}\right)=2 d$.

As we introduce in the first section, there are some results to find the spectral radius (largest eigenvalue) of nonnegative irreducible tensors. Here, we also give two theorems on the minimum and the maximum characterizations of the spectral radius of nonnegative weakly irreducible tensors.

Theorem 3.5. Let $\mathcal{A}=\left(a_{i_{1} i_{2} \ldots i_{m}}\right) \in \mathbb{R}_{+}^{[m, n]}$ be a weakly irreducible tensor. Then

$\min _{x \in \mathbb{R}_{++}^{n}} \max _{\gamma \in C\left(G_{\mathcal{A}}\right)}\left(\prod_{i \in \gamma} \frac{\left(\mathcal{A} x^{m-1}\right)_{i}}{x_{i}^{m-1}}\right)^{\frac{1}{|\gamma|}}=\rho(\mathcal{A})=\max _{x \in \mathbb{R}_{++}^{n}} \min _{\gamma \in C\left(G_{\mathcal{A}}\right)}\left(\prod_{i \in \gamma} \frac{\left(\mathcal{A} x^{m-1}\right)_{i}}{x_{i}^{m-1}}\right)^{\frac{1}{|\gamma|}}$.

Proof. Let $\mathcal{B}=X^{-(m-1)} \mathcal{A} X$, where the matrix $X=\operatorname{diag}\left(x_{1}, x_{2}, \ldots, x_{n}\right)$, $x_{i}>0, i=1,2, \ldots, n$. It is easy to see $\mathcal{B}$ is nonnegative weakly irreducible, and $G_{\mathcal{A}}$ and $G_{\mathcal{B}}$ are the same digraph. So by Theorem 3.1, we have

$$
\min _{\gamma \in C\left(G_{\mathcal{A}}\right)}\left(\prod_{i \in \gamma} K_{i}(\mathcal{B})\right)^{\frac{1}{|\gamma|}} \leqslant \rho(\mathcal{B}) \leqslant \max _{\gamma \in C\left(G_{\mathcal{A}}\right)}\left(\prod_{i \in \gamma} K_{i}(\mathcal{B})\right)^{\frac{1}{|\gamma|}} .
$$

Calculation gives that

$$
K_{i}(\mathcal{B})=\frac{1}{x_{i}^{m-1}} \sum_{i_{2}, \cdots, i_{m}=1}^{n} a_{i i_{2} \ldots i_{m}} x_{i_{2}} \ldots x_{i_{m}}=\frac{\left(\mathcal{A} x^{m-1}\right)_{i}}{x_{i}^{m-1}},
$$

and since Lemma 2.3 shows that $\rho(\mathcal{A})=\rho(\mathcal{B})$, we obtain

$$
\min _{\gamma \in C\left(G_{\mathcal{A}}\right)}\left(\prod_{i \in \gamma} \frac{\left(\mathcal{A} x^{m-1}\right)_{i}}{x_{i}^{m-1}}\right)^{\frac{1}{\gamma \gamma}} \leqslant \rho(\mathcal{A}) \leqslant \max _{\gamma \in C\left(G_{\mathcal{A}}\right)}\left(\prod_{i \in \gamma} \frac{\left(\mathcal{A} x^{m-1}\right)_{i}}{x_{i}^{m-1}}\right)^{\frac{1}{\gamma \gamma}} .
$$

When $\left(x_{1}, x_{2}, \ldots, x_{n}\right)^{\mathrm{T}}$ is a positive eigenvector of $\mathcal{A}$ corresponding to $\rho(\mathcal{A})$, the equalities in the above equation hold, so we can get

$$
\min _{x \in \mathbb{R}_{++}^{n}} \max _{\gamma \in C\left(G_{\mathcal{A}}\right)}\left(\prod_{i \in \gamma} \frac{\left(\mathcal{A} x^{m-1}\right)_{i}}{x_{i}^{m-1}}\right)^{\frac{1}{|\gamma|}}=\rho(\mathcal{A})=\max _{x \in \mathbb{R}_{++}^{n}} \min _{\gamma \in C\left(G_{\mathcal{A}}\right)}\left(\prod_{i \in \gamma} \frac{\left(\mathcal{A} x^{m-1}\right)_{i}}{x_{i}^{m-1}}\right)^{\frac{1}{|\gamma|}} .
$$


By the proof of the above theorem, we get the following result.

Theorem 3.6. Let $\mathcal{A}=\left(a_{i_{1} i_{2} \ldots i_{m}}\right) \in \mathbb{R}_{+}^{[m, n]}$ be a weakly irreducible tensor. Then

$$
\min _{\gamma \in C\left(G_{\mathcal{A}}\right)}\left(\prod_{i \in \gamma} \frac{\left(\mathcal{A} x^{m-1}\right)_{i}}{x_{i}^{m-1}}\right)^{\frac{1}{|\gamma|}} \leqslant \rho(\mathcal{A}) \leqslant \max _{\gamma \in C\left(G_{\mathcal{A}}\right)}\left(\prod_{i \in \gamma} \frac{\left(\mathcal{A} x^{m-1}\right)_{i}}{x_{i}^{m-1}}\right)^{\frac{1}{|\gamma|}}
$$

where $x=\left(x_{1}, x_{2}, \ldots, x_{n}\right)$ is a positive vector.

From Theorem 3.3 , the following result can be obtained.

Theorem 3.7. Let $\mathcal{A} \in \mathbb{R}_{+}^{[m, n]}$ be a weakly irreducible tensor. Let the girth of $G_{\mathcal{A}}$ is $g$. Then

$$
\min _{X \in D^{n}}\left(\prod_{i=n-g+1}^{n} K_{i}\left(X^{-(m-1)} \mathcal{A} X\right)\right)^{\frac{1}{g}}=\rho(\mathcal{A})=\max _{X \in D^{n}}\left(\prod_{i=1}^{g} K_{i}\left(X^{-(m-1)} \mathcal{A} X\right)\right)^{\frac{1}{g}},
$$

where the slice sums of tensor $X^{-(m-1)} \mathcal{A} X$ are in the order $K_{1} \leqslant K_{2} \leqslant \cdots \leqslant$ $K_{n}$ and $D^{n}$ is the set of all the $n \times n$ positive diagonal matrices.

Remark. Brualdi gives the characterizations of the spectral radius of a nonnegative irreducible matrices with all diagonal entries zero ( Corollary 4.10 and 4.11 of [2]). Theorem 3.5 and 3.7 generalize them to general nonnegative weakly irreducible tensors without the condition that diagonal entries are zero.

\section{References}

\section{References}

[1] R.A. Brualdi, H.J. Ryser, Combinatorial Matrix Theory, Cambridge University Press, Cambridge, 1991.

[2] R.A. Brualdi, Matrices, eigenvalues, and directed graphs, Linear and Multilinear Algebra 11(1982) 143-165.

[3] C. Bu, Y. Wei, L. Sun, J. Zhou, Brualdi-type eigenvalue inclusion sets of tensors, Linear Algebra Appl. http://dx.doi.org/10.1016/j.laa.2015.04.034. 
[4] J. Cooper, A. Dutle, Spectra of uniform hypergraphs, Linear Algebra Appl. 436 (2012) 3268-3292.

[5] K. Chang, K. Pearson, T. Zhang, Perron Frobenius Theorem for nonnegative tensors, Commun. Math. Sci. 6(2008) 507-520.

[6] K. Chang, K. Pearson, T. Zhang, Primitivity, the convergence of the NQZ method, and the largest eigenvalue for nonnegative tensors, SIAM J. Matrix Anal. Appl. 32(2011) 806-819.

[7] Z. Chen, L. Qi, Q. Yang, Y. Yang, The solution methods for the largest eigenvalue (singular value) of nonnegative tensors and convergence analysis, Linear Appl. 439(2013) 3713-3733.

[8] S. Friedland, S. Gaubert, L. Han, Perron-Frobenius theorem for nonnegative multilinear forms and extensions, Linear Algebra Appl. 438(2013) 738-749.

[9] S. Hu, L. Qi, The Laplacian of a uniform hypergraph, J Comb Optim 29(2015) 331-366.

[10] S. Hu, L. Qi, J. Xie, The largest Laplacian and signless Laplacian Heigenvalues of a uniform hypergraph, Linear Algebra Appl. 469(2015) $1-27$.

[11] L.H. Lim, Singular values and eigenvalues of tensors: a variational approach, in: Proceedings 1st IEEE International Workshop on Computational Advances of Multitensor Adaptive Processing (2005) 129-132.

[12] M. Ng, L. Qi, G. Zhou, Finding the largest eigenvalue of a nonnegative tensor, SIAM J. Matrix Anal. Appl. 31(2009) 1090-1099.

[13] Q. Ni, L. Qi, A quadratically convergent algorithm for finding the largest eigenvalue of a nonnegative homogeneous polynomial map, Journal of Global Optimization (61)(4)2015 627-641.

[14] K. Pearson, T. Zhang, On spectral hypergraph theory of the adjacency tensor, Graphs and Combin. 30(2014) 1233-1248.

[15] L. Qi, Eigenvalues of a real supersymmetric tensor, J. Symbolic Comput. 40(2005) 1302-1324. 
[16] Li. Qi, $H^{+}$-Eigenvalues of laplacian signless laplacian tensors, Commun. Math. Sci. 12(2014) 1045-1064.

[17] J. Shao, A general product of tensors with applications, Linear Algebra Appl. 439(2013) 2350-2366.

[18] J, Shao, H. Shan, B. Wu, Some spectral properties and characterizations of connected odd-bipartite uniform hypergraphs, Linear and Multilinear Algebra DOI:10.1080/03081087.2015.1009061.

[19] Y. Yang, Q. Yang, Further results for PerronCFrobenius theorem for nonnegative tensors, SIAM. J. Matrix Anal. Appl. 31(2010) 2517-2530.

[20] Y. Yang, Q. Yang, Geometric simplicity of the spectral radius of nonnegative irreducible tensors, Front. Math. China 8(1)(2013) 129-140.

[21] G. Zhou, L. Caccetta, L. Qi, Convergence of an algorithm for the largest singular value of a nonnegative rectangular tensor, Linear Algebra Appl. 438 (2013) 959-968.

[22] J. Zhou, L. Sun, W. Wang, C. Bu, Some spectral properties of uniform hypergraphs, Electron. J. Combin. 21 (2014) P4.24.9 